\title{
Are We Heading Towards a Thirsty Planet
}

\author{
Shangrun $\mathrm{Yao}^{1, \text { a }}$ \\ ${ }^{1}$ School of Electrical and Electric Engineering, North China Electric Power University, Beijing, \\ 102206, China \\ ayaoshangrun@163.com
}

Keywords: The Systems Dynamics, Intervention

\begin{abstract}
Water is the important substantial foundation of social and economic development. In view of its importance, establishing an appropriate model to predict the supply and demand of water in the future is vitally necessary. By analyzing the future situation, we can take some intervention measures as early as possible. Firstly, we build a simulation system model based on the Systems Dynamics (SD). There are two reasonable indicators, including the amount of the primary balance (PB) and the amount of the secondary balance (SB), to reflect the relation between the supply and demand of water. $\mathrm{PB}$ reflects the water shortage degree and SB reflects the dependent degree on the water resources in other regions. Based on 48 variables and four subsystems, the SD model can accurately and dynamically simulate the change of the real system. Then, we propose an intervention, it mainly contains two aspects. One is to reduce the exploitation of the groundwater, the other one is to strengthen management and increase the advanced equipment.
\end{abstract}

\section{Introduction}

Water resources system is a dynamical feedback, complex and time-varying system related to social、economic、environmental、ecological and other fields. Usually there is interconnectedness and mutual influence among system elements and meanwhile the elements are variable with time. In order to measure the ability of a region to provide clean water to meet the needs of its population, we design system dynamics model (SD) and build four subsystems in the model to analyze the water resources' supply and requirement contradiction and the interrelation of the dependence and restriction of the subsystems. In addition, we establish two reasonable indicators including the amount of the primary balance and the amount of the secondary balance to measure whether the water supply-demand is in a state of balance. Moreover, we can predict what the situation will be in 15 years by the model.

\section{SD Model}

Step1: Determine the system boundaries

System boundaries are not only the distinguished boundaries between the system and its environment but also an important medium of interaction with its environment. Only after determining the system boundary, can we define an effective structure and parameter. Generally we choose the area that we study as the system boundary.

Step2: Construct subsystems and Analyze causal connections

By learning related knowledge, we construct four subsystems and analyze the causal connections among the factors that we have considered. The four subsystems are the water demand subsystem, the natural water resources subsystem, the artificial water renewable subsystem and the exotics water subsystem. Also, we classify the variables into three types: state variables, speed variables and instrumental variables for the convenience of the following work. We can obtain the trend of some speed variables' change by nonlinear fitting. The results of the classification are presented in the below figure. 


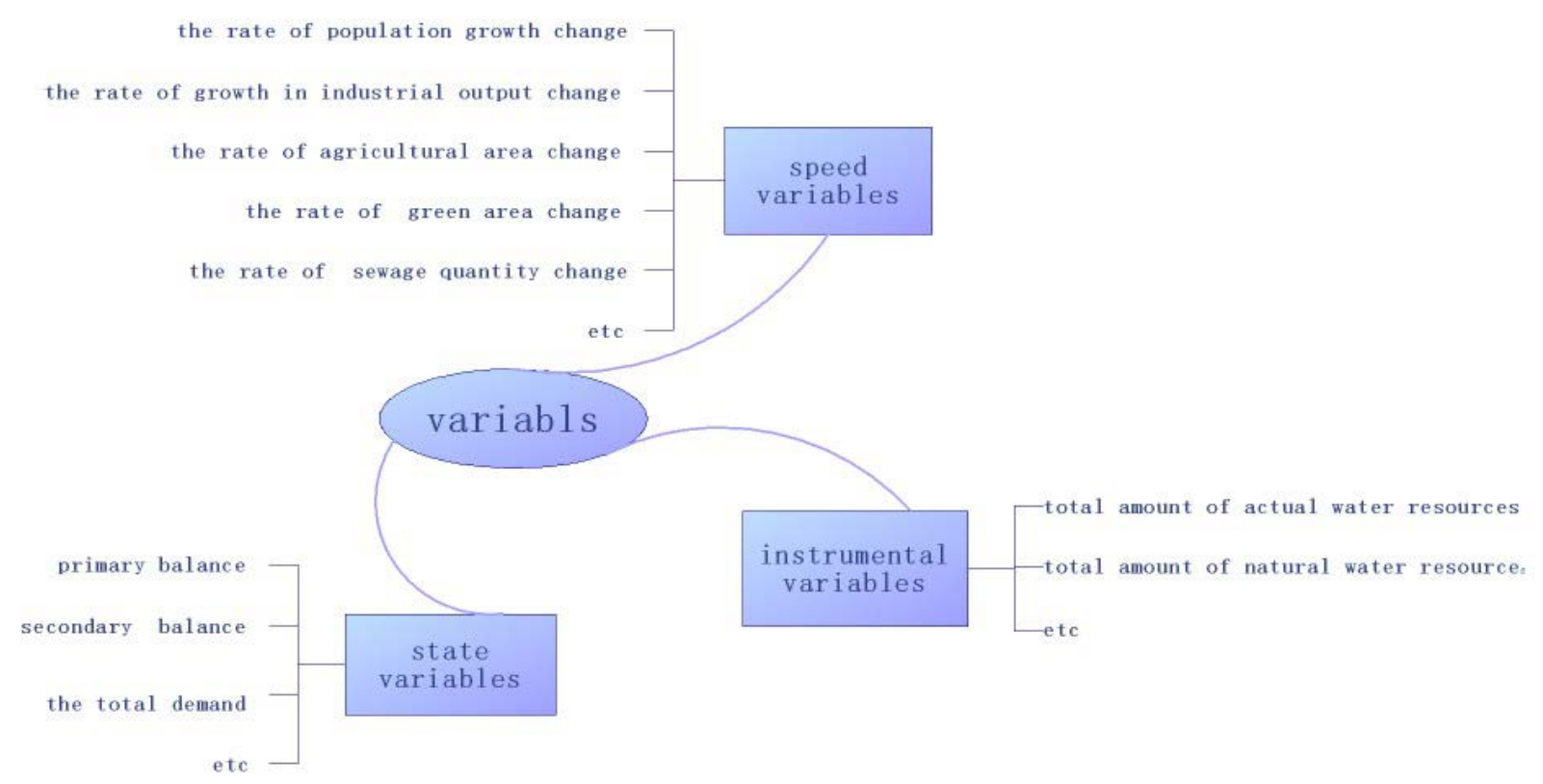

Figure1: the classification of the variables

Step3: Build the SD diagram

Before simulating our model, we should build the stock and flow diagram first. The stock and flow diagram is as follows.

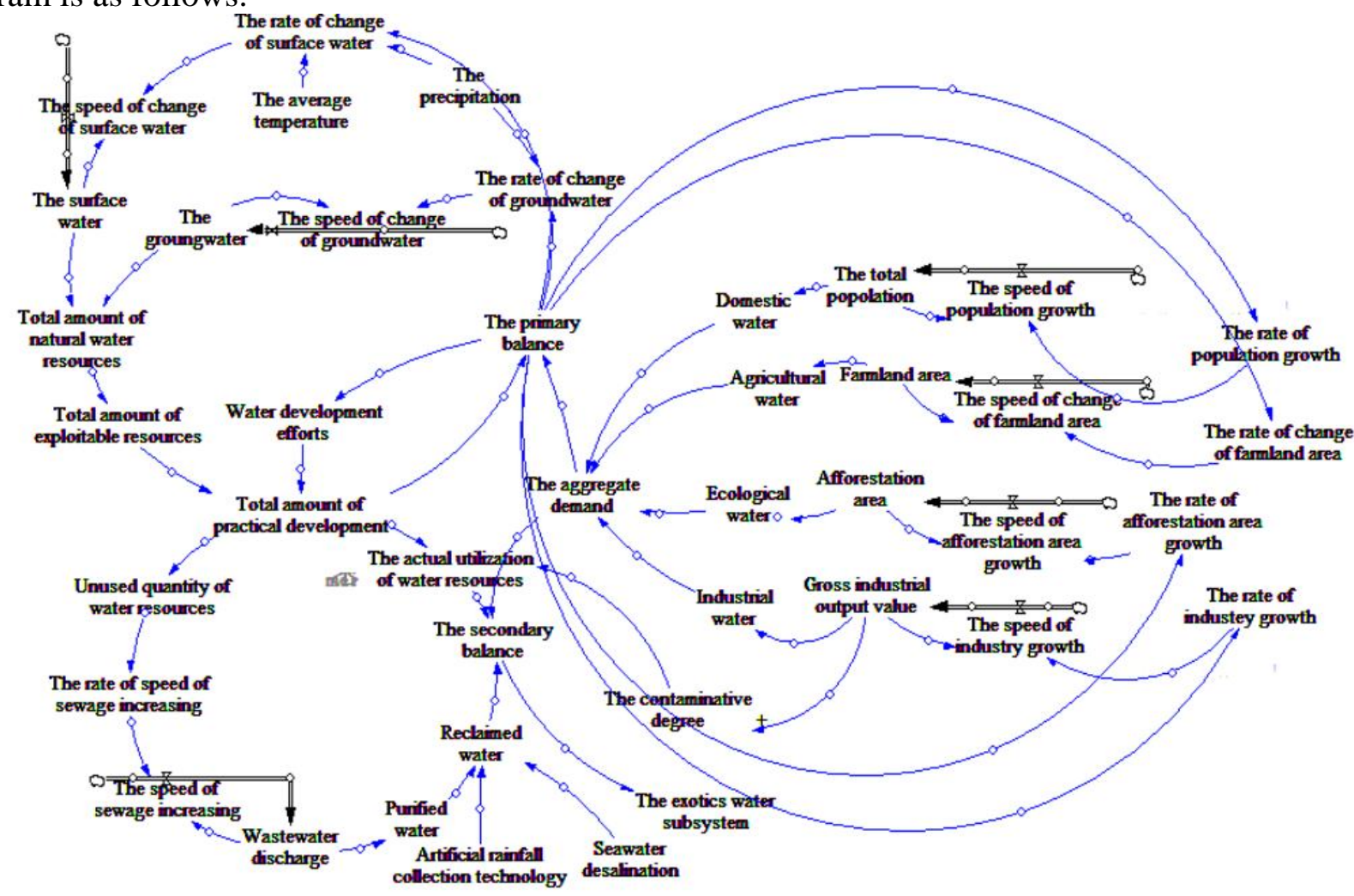

Step4: Establish equations

Figure2: the stock and flow diagram

We establish equations according to the SD diagram and it essentially uses the continuous model to substitute for the discrete model, then we only need to simulate the continuous model. The main structural equations of the system are listed in the following table. 
Table1: the main structural equations of the system

\section{Number Structure equation}

(1)

The surface water=INTEG(The speed of change of surface water,191)

(2) Total amount of natural water resources=The surface water+The ground water

(3) Waste water discharge=INTEG(The speed of sewage increasing,514423)

(4) Farmland area=INTEG(The speed of change of farmland area,1.10379e+007)

The aggregate demand $=\mathrm{A}+\mathrm{I}+\mathrm{E}+\mathrm{D}$

(6) Agricultural water=Farmland area*1.42e-005

(7) Purified water $=$ Waste water discharge $* 1.5 \mathrm{e}-005$

The groundwater=INTEG(The speed of change of groundwater,100)

Step5: Calculate and analyze

By running Vensim, we can calculate the amount of the primary balance and the amount of the secondary balance in 15 years.

Step6: Examine the model

Firstly, we examine the model's rationality and validity by two ways. One is to use part of the data we have gathered to predict the other part, the other one is to use other methods such as neutral network model to forecast and compare the results.

Secondly, we examine the model's sensitivity. We can observe the behavior of the system by changing the value of the variables to judge the stability of the system.

\section{Intervention}

Here we classify the intervention plan into physical intervention and economic intervention, which correspond to physical scarcity and economic scarcity.

Physical intervention

The main concern is focused on the little volume of all the natural water. Natural factors cause the shortage of water resources in some degree whether the precipitation or the total amount of surface water and groundwater is less than the national average. In order to make up for physical scarcity, we provide solutions as follows and they aim at exploring new resources of water.

Desalinize the seawater.

Seawater desalination and direct seawater utilization should be encouraged and supported. Some cities belong to coastal area, so improving the rate of seawater desalination can increase the total amount of available fresh water.

Advanced technology can be developed to increase rainfall collection rate.

Rainfall is the other water resource, like seawater desalination, rainfall collection can also reduce the burden of water supply.

Implement water transmission and conservancy projects(including trans-regional water transmission project such as the South-to-North water diversion project 、 fresh water conservancy project, etc.)

Through this way, there is hope to achieve solving the problem of uneven and unreasonable allocation of water resources effectively.

Make artificial rainfall when in dry years.

Use artificial means to reduce the effects of drought.

Introduce a massive afforestation program. 
Forests can improve the ecological environment to regulate climate efficiently and prevent the occurrence of natural disasters.

Economic intervention

Economic scarcity is the result of poor management and lack of infrastructure and it limits the availability of clean water. The problems center on four aspects: industrial waste, agricultural waste, pollution and others. So we provide corresponding solutions and we think that the economic interventions can decrease water waste and consumptions.

Increase the efficiency of the usage of the water

There are many measures to improve water use efficiency. For example, highly polluted water could be used for navigation、 purify and recycle the sewage vadopt interval drip irrigation mode to irrigate in agriculture, etc.

Transform to water-saving industries

Improve existing laws, regulations and policies to protect water sources and strengthen management

Reduce water pollution

Here ,we can limit the use of pesticides, stop people from throwing rubbish into the water, prohibit factories from discharging sewage indiscriminately, etc.

The above is the intervention plan we designed for Shandong province. Of course, our plan will have effects on the surrounding area and the entire aquatic ecosystem. For example, making artificial rainfall and painting trees may change the climate of local region. Besides, start new water transportation line can narrow the difference between the North and the South.

\section{Conclusion}

We can use the simulation system model based on the Systems Dynamics to predict the supply and demand of water in the future efficiently. By analyzing the future situation, we can take some intervention measures like what we refer to above as early as possible.

\section{References}

[1] Wenhua Li, Sustainable development and resource strategies, Editorial Board, Natural Resources, Beijing 100039, China R512.8 A 1007-8134(2014)04-000-6.

[2] Zhou Hou-qing, XU You-zhuan, Water Resources Assessment for China, Beijing 100069, China Water Power Press.

[3] Hu Huang, Zhuoru Chen, Danqing Wang, Thinking of solving the problem of Water shortage in coastal area.

[4] Dongchun Ma, Study on the water resources shortage causes and solutions in Shandong Province. 